\title{
A Game-Theoretic Approach to Joint Rate and Power Control for Uplink CDMA Communications
}

\author{
Madhusudhan R. Musku, Anthony T. Chronopoulos, Senior Member, IEEE, Dimitrie C. Popescu, Senior \\ Member, IEEE, and Anton Ştefănescu
}

\begin{abstract}
Next generation wireless systems will be required to support heterogeneous services with different transmission rates that include real time multimedia transmissions, as well as non-real time data transmissions. In order to provide such flexible transmission rates, efficient use of system resources in next generation systems will require control of both data transmission rate and power for mobile terminals. In this paper we formulate the problem of joint transmission rate and power control for the uplink of a single cell CDMA system as a noncooperative game. We assume that the utility function depends on both transmission rates and powers and show the existence of Nash equilibrium in the non-cooperative joint transmission rate and power control game (NRPG). We include numerical results obtained from simulations that compare the proposed algorithm with a similar one which is also based on game theory and it also updates the transmission rates and powers simultaneously in a single step.
\end{abstract}

Index Terms-Power control, rate control, non-cooperative games, Nash equilibrium.

\section{INTRODUCTION}

$\mathbf{N}$ EXT generation wireless systems will provide a wide range of services for mobile users, from multimedia transmissions performed in real time to transmission of data that can tolerate delay and which is not performed in real time. In order to provide these heterogeneous services, efficient use of system resources in this case requires control of both transmission data rate and power. This requirement implies the need for joint rate and power control for mobile terminals, as opposed to only power control that is currently performed in wireless systems [1], [3], [4], [7]-[9], [13], [14], [16].

The main goal of power control is to provide adequate quality for the signal of each mobile terminal at the receiver without causing unnecessary interference to signals transmitted by other mobile terminals. Power control helps also to extend the battery life of mobile terminals by ensuring

Manuscript received May 7, 2007, revised April 2, 2008, January 16, 2009, and August 16, 2009; accepted September 3, 2009. The associate editor coordinating the review of this paper and approving it for publication was Prof. Kin Leung.

This work was supported in part by National Science Foundation under grant CCR-0312323.

M. Musku was with the Department of Electrical and Computer Engineering, University of Texas at San Antonio.

A. T. Chronopoulos is with the Department of Computer Science, University of Texas at San Antonio. E-mail: atc@cs.utsa.edu.

D. C. Popescu is with the Department of Electrical and Computer Engineering, Old Dominion University, 231 Kaufman Hall, Norfolk, VA 23529. E-mail: dpopescu@odu.edu.

Anton Ştefănescu is with the Faculty of Mathematics and Informatics, University of Bucharest, Romania.

Digital Object Identifier that they transmit at the minimum power level necessary to achieve the required QoS. Game-theoretic approaches to power control [1], [3], [4], [13] describe QoS for mobile terminals using utility functions. For power control the utility function for a given terminal depends usually on both the SIR and the transmission power of the terminal. In [3], [13] the utility function depends on low-level system parameters like modulation, coding, and packet size. In [4] a different utility function which depends on the channel capacity and decouples lower layer decisions like modulation and coding is proposed.

Research in the area of joint rate and power control is emerging and several algorithms have been proposed by researchers. These can be classified as centralized algorithms, such as those in [6], [11], or distributed algorithms like the ones in [2], [5], [15]. In the category of centralized algorithms the approach in reference [6] uses a different model than the one considered in our paper and is not based on game theory, while the one in [11] uses a single utility function for the system called the "system figure of merit" and deals with a dynamic scenario where some parameters of the links change during the simulations. In the distributed algorithms category, the game theoretic approach in [15] uses a similar CDMA system model as the one considered in our paper, and the proposed algorithm for joint rate and power control updates powers and rates in a similar fashion to our proposed approach, that is jointly in a single step, which results in terminals closer to the base station achieving higher rates at lower powers while terminals farther away from the base station transmit at full power with low rates. The algorithm in [5] uses a two-layered game in which the first game determines a set of rates and the second game determines the powers. This is different than our approach where powers and rates are computed jointly in a single game. The game-theoretic approach in [2] solves the problem of joint rate and power allocation using discrete link adaptation. The system model in [2] is focused explicitly on GPRS technology where discrete options for the data rate are specified through specific parameters of the modulation scheme.

In our paper we consider the uplink of a single cell CDMA system and the utility function defined in [13], but assume that each user is capable of variable transmission rates in addition to transmit powers. We formulate a NRPG which determines the optimal rate of transmission and allocates the power required for transmission based on utility maximization. We discuss existence of Nash equilibria for the NRPG and present an algorithm which reaches a Nash equilibrium in a distributed manner by updating transmission rates and powers 
jointly in a single step similar to [15]. We present numerical results obtained from simulations of the NRPG algorithm which illustrate its convergence to a Nash equilibrium and compares it with the one obtained using the approach in [15]. We note that a comparison with the alternative approaches in [2], [5] is not meaningful due to the significant differences in either the system model in the case of [2] or the update procedure in the case of [5]. We also note that we do not consider the problem of any existing users leaving (or of new users entering) the network. This is an admission control problem which is beyond the scope of the paper and was not considered in any of the aforementioned game-theoretic approaches to power control [13] or joint rate and power control [2], [5], [15].

The paper is organized as follows: in Section II, we describe the system model, and we introduce the utility function to be used for joint rate and power control. In Section III, we formulate joint rate and power control as a non-cooperative game (NRPG), and we investigate the existence of a Nash equilibrium solution for this game. In Section IV, we formally state the joint rate and power control algorithm and discuss its convergence. In Section V, we present numerical results obtained from simulations, and compare the proposed algorithm with the algorithm in [15]. Final conclusions are presented in Section VI.

\section{System Model And Utility Function}

We consider the uplink communication of a single-cell CDMA wireless system with $N$ mobile terminals (users) that transmit data to the base station, for which the SIR corresponding to a given user $j$ is [13]

$$
\gamma_{j}=\frac{W}{r_{j}} \frac{h_{j} p_{j}}{\sum_{k=1, k \neq j}^{N} h_{k} p_{k}+\sigma^{2}} \quad j=1, \ldots, N
$$

where $W$ is the available bandwidth, $h_{j}$ is the path gain of user $j$ to the base station, $r_{j}$ is the transmission rate, $p_{j}$ is power of user $j$ and $\sigma^{2}$ is the power spectral density of the additive white Gaussian noise (AWGN) which causes degradation of the the received signal at the base station. This expression assumes that users in the CDMA system are assigned pseudorandom noise (PN) sequences, and that conventional matched filter detectors are used at the receiver [13]. We note that because the available bandwidth is shared by all users, the transmission of any user in the system creates interference to the other users' transmissions, and the SIR defined in equation (1) serves as a measure for the QoS.

In order to achieve satisfactory QoS as implied by transmission with given rate and SIR values, users should transmit with the minimum power necessary to achieve these values. This will minimize energy consumption and will help increase the battery life for mobile terminals. The level of satisfaction of a given user $i$ with SINR $\gamma_{i}$ that transmits at rate $r_{i}$ using power $p_{i}$ can be quantified by using utility functions. The concept of utility (or payoff) is commonly used in microeconomics and game theory to denote the level of satisfaction of a decisionmaker with specific products or services as a result of its actions [12]. In wireless systems utility functions are typically related to the user SIR, as well as to the transmission rate and power, and several utility functions have been used for power and rate control [3]-[6], [11], [13], [15].

In the case of power control for wireless data transmission utility functions must satisfy the following properties [13]:

- For fixed transmit powers, the utility increases with the increase in the SIR of the terminal.

- For fixed SIR, the utility decreases as the transmitted power increases.

For rate control utility functions must satisfy similar properties:

- For fixed transmission rates, the utility increases with the increase in the SIR of the terminal.

- For fixed SIR, the utility increases as the rate increases.

These properties of the utility functions are illustrated in the plots in Figure 1. As SIR increases, the terminal experiences low error rates and this leads to efficient utilization of system resources like the mobile terminals' battery drain [13]. The asymptotic increase in utility in the high SIR region is due to the fact that for sufficiently large SIR values, the error rate approaches zero [13]. Figure 2 (a) shows that for fixed interference, the utility increases initially as power increases and then it decreases for higher power values because the terminal consumes more power. The utility function behaves in a similar way for varying transmission rates as seen in Figure 2 (b).

In our game theoretic approach to joint rate and power control we consider the expression for the utility function of a given user $j$ as in [13], but assume that the users have a variable rate

$$
u_{j}\left(r_{j}, p_{j}\right)=\frac{L r_{j} f\left(\gamma_{j}\right)}{M p_{j}}[\mathrm{bits} / \mathrm{J}] \quad j=1, \ldots, N
$$

where $f\left(\gamma_{j}\right)$ is the efficiency function defined as

$$
f\left(\gamma_{j}\right)=\left(1-2 P_{e}\right)^{M}
$$

where $P_{e}$ is the bit error rate (BER), $L$ is the number of information bits in a packet of length $M$. We consider Noncoherent FSK modulation for which the BER is given as $P_{e}=$ $\frac{1}{2} e^{-\gamma / 2}$. We note that we can express $\gamma_{j}$ in terms of $r_{j}$ and $p_{j}$ as

$$
\gamma_{j}=c_{j} \frac{p_{j}}{r_{j}}
$$

where $c_{j}$ does not depend on $r_{j}$ and $p_{j}$, is given as

$$
c_{j}=\frac{W h_{j}}{\sum_{k=1, k \neq j}^{N} h_{k} p_{k}+\sigma^{2}}
$$

In this context, the objective of each user in the system is to adapt its transmitted power and rate in a distributed manner, such that its corresponding utility is maximized. 


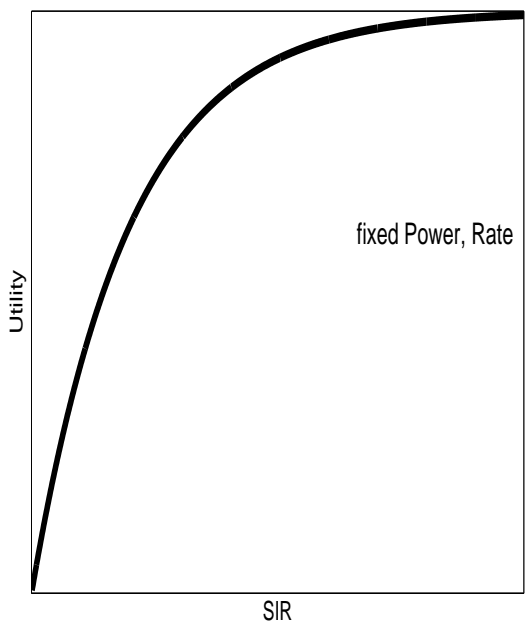

(a) Utility vs. SIR

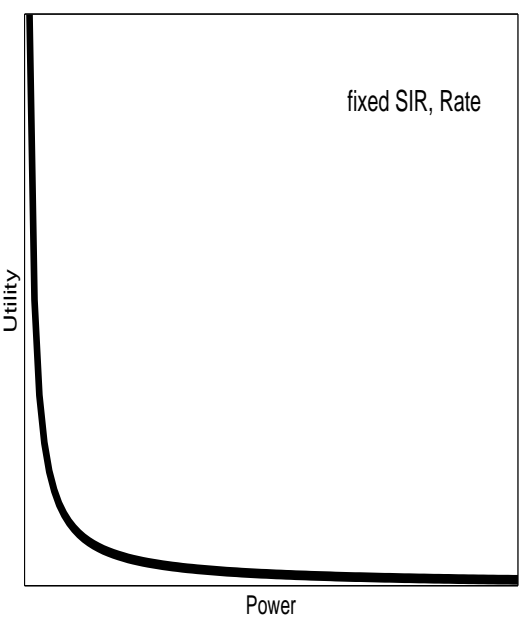

(b) Utility vs. Power

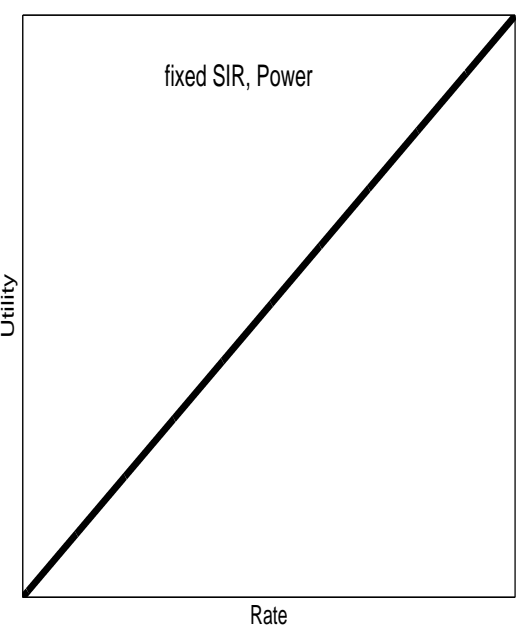

(c) Utility vs. Rate

Fig. 1. The behavior of utility functions for increasing SIR with fixed power and rate, increasing power with fixed SIR and rate, increasing rate with fixed power and SIR

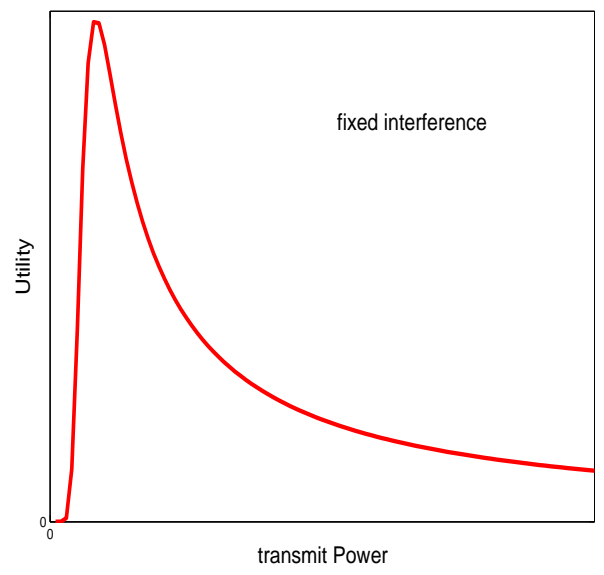

(a)

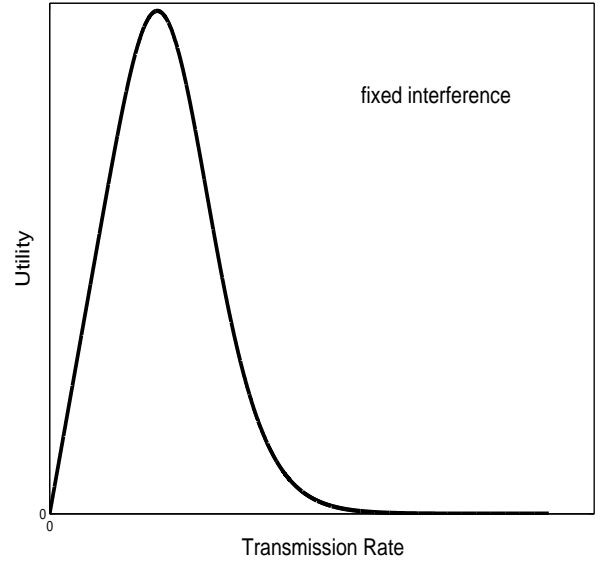

(b)

Fig. 2. Shape of the utility as a function of the user transmission rate and power for fixed interference.

\section{Formulation as a Non-Cooperative Game}

In a non-cooperative game for rate and power control each user adjusts rate $r_{j}$ and power $p_{j}$ in order to maximize its corresponding utility $u_{j}$. The NRPG is defined by

$$
\max _{r_{j} \in R_{j}, p_{j} \in P_{j}} u_{j}(\mathbf{r}, \mathbf{p}) \quad j=1, \ldots, N
$$

where $u_{j}$ is the user utility function in (2) and $R_{j}, P_{j}$ are the strategy spaces of user $j$. We note that $u_{j}$ is not defined for either $r_{j}=0$ or $p_{j}=0$. Moreover $u_{j}$ as a function of $\left(r_{j}, p_{j}\right)$ has no limit at $(0,0)$. So we consider strategy spaces as $R_{j}=\left(0, \bar{r}_{j},\right]$ and $P_{j}=\left(0, \bar{p}_{j},\right]$, which are convex sets defined in terms of minimum and maximum rates and powers. Formally we define the NRPG as $G=\left[\mathcal{N},\left\{P_{j}, R_{j}\right\},\left\{u_{j}\right\}\right]$ with $\mathcal{N}=\{1,2, \ldots, N\}$ being the index set for the active users in the cell, such that each user $j$ selects a rate $r_{j} \in$ $R_{j}$ and a power $p_{j} \in P_{j}$ to maximize utility function $u_{j}$, where $P_{j}$ represents user $j$ 's strategy set in powers and $R_{j}$ represents user $j$ 's strategy set in rates. Let the rate vector $\mathbf{r}=\left(r_{1}, r_{2}, \ldots, r_{N}\right)^{T} \in R=R_{1} \times R_{2} \times \ldots \times R_{N}$, and power vector $\mathbf{p}=\left(p_{1}, p_{2}, \ldots, p_{N}\right)^{T} \in P=P_{1} \times P_{2} \times \ldots \times P_{N}$ (where $T$ represents the transpose operator, $R_{j}=\left(0, \bar{r}_{j}\right]$ and
$\left.P_{j}=\left(0, \bar{p}_{j}\right]\right)$ denote the outcome of the game in terms of selected rates and powers for all users.

\section{A. The Nash Equilibrium for Individual Rate and Power Games}

A Nash equilibrium is a fixed point of a non-cooperative game where no user can increase the value of its utility function through individual action. For joint rate and power control we investigate Nash equilibrium solutions for the rate and power games defined in the previous section. We will show that a Nash equilibrium solution in both transmission rates and transmit powers exists. Next, we will show that a Nash equilibrium exists in the problem of joint transmission rates and transmit powers.

First, we will consider that the players wish to maximize their utilities only with respect to transmission rates. For a given power vector $\mathbf{p}$, the utility function $u_{j}$ of user $j$ is defined on $R$ and the game is formally represented as $G(\mathbf{p})=$ $\left[\mathcal{N},\left\{R_{j}\right\},\left\{u_{j}(., \mathbf{p})\right\}\right]$.

Then, a Nash equilibrium in rates is formally defined as

Definition 1: A rate vector $\mathbf{r}=\left(r_{1}, \ldots, r_{N}\right)$ is a Nash equilibrium of the NRPG $G(\mathbf{p})$ if, for every $j \in \mathcal{N}$, 
$u_{j}\left(r_{j}, \mathbf{r}_{-j}, \mathbf{p}\right) \geq u_{j}\left(r_{j}, \mathbf{r}_{-j}, \mathbf{p}\right)$ for all $r_{j} \prime \in R_{j}$, where $\mathbf{r}_{-j}=\left(r_{1}, \ldots, r_{j-1}, r_{j+1}, \ldots, r_{N}\right)^{T}$ is the $N-1$ dimensional vector of user rates that does not contain user $j$ 's rate.

In fact, the game $G(\mathbf{p})$ is a "dummy game", since user $j$ 's utility function depends only upon his own strategy $r_{j}$. Then a Nash equilibrium is formed by any set of $N$ maximizing strategies of the $N$ users. This is noteworthy because each user has only one maximizing strategy in his strategy set.

We next present two results similar to [13] that prove the existence of Nash equilibrium in the case of fixed power (rate) and variable transmission rate (power). These results are similar to [13], but they are proved in a simpler way.

Proposition 1: For each $\mathbf{p} \in P$, there is a unique maximum point of $u_{j}$ in $R_{j}$. (The game $\left[\mathcal{N},\left\{R_{j}\right\},\left\{u_{j}(., \mathbf{p})\right\}\right]$ has a unique Nash equilibrium.)

Proof: Fixing p, write simply $u_{j}\left(r_{j}\right)$ for $u_{j}\left(r_{j}, \mathbf{r}_{-j}, \mathbf{p}\right)$.

We can show that $u_{j}($.$) has a unique maximizing point \hat{r}_{j}$ on $(0, \infty)$ and

(a) $\hat{r}_{j} \in\left(0, \frac{M p_{j} c_{j}}{2(M-1)}\right)$

(b) $u_{j}$ strictly increases on $\left(0, \hat{r}_{j}\right)$

(c) $u_{j}$ strictly decreases on $\left(\hat{r}_{j}, \infty\right)$

The derivative of $u_{j}$ with respect to $r_{j}$ is,

$$
\frac{\partial u_{j}}{\partial r_{j}}=\frac{L}{M p_{j}}\left(1-e^{-\frac{p_{j} c_{j}}{2 r_{j}}}\right)^{M-1} \phi\left(r_{j}\right)
$$

where

$$
\phi\left(r_{j}\right)=1-e^{-\frac{p_{j} c_{j}}{2 r_{j}}}-\frac{M p_{j} c_{j}}{2 r_{j}} e^{-\frac{p_{j} c_{j}}{2 r_{j}}}
$$

Since the first factor is positive, the sign of (6) is given by $\phi(j)$. We have

$$
\frac{\partial \phi\left(r_{j}\right)}{\partial r_{j}}=\frac{1}{r_{j}^{2}} e^{-\frac{p_{j} c_{j}}{2 r_{j}}}\left(\frac{-p_{j} c_{j}}{2}+\frac{M p_{j} c_{j}}{2}-\frac{M^{2} p_{j}^{2} c_{j}^{2}}{4 r_{j}}\right)
$$

hence:

$$
\frac{\partial \phi\left(r_{j}\right)}{\partial r_{j}}\left\{\begin{array}{l}
<0, \quad \text { if } \quad r_{j} \in\left(0, \frac{M p_{j} c_{j}}{2(M-1)}\right) \\
=0, \quad \text { if } \quad r_{j}=\frac{M p_{j} c_{j}}{2(M-1)} \\
>0, \quad \text { if } \quad r_{j} \in\left(\frac{M p_{j} c_{j}}{2(M-1)}, \infty\right)
\end{array}\right.
$$

Since $\lim _{r_{j} \rightarrow 0} \phi\left(r_{j}\right)=1$, and $\phi\left(\frac{M p_{j} c_{j}}{2(M-1)}\right)=1-M e^{\frac{1-M}{M}}<$ 0 , it follows that $\phi\left(r_{j}\right)$ has a zero in the interval $\left(0, \frac{M p_{j} c_{j}}{2(M-1)}\right)$ (denoted by $\hat{r}_{j}$ ), and changes the sign from plus to minus at this point. Hence, we will have (a) and (b). Further, since $\lim _{r_{j} \rightarrow \infty} \phi\left(r_{j}\right)=0$, and $\phi\left(r_{j}\right)$ increases on $\left(\frac{M p_{j} c_{j}}{2(M-1)}, \infty\right)$, it follows that $\phi\left(r_{j}\right)$ is negative in this interval. Hence $\phi\left(r_{j}\right)$ is negative on $\left(\hat{r}_{j}, \infty\right)$, which implies (c).

It follows that the unique maximizing point of $u_{j}$ in the domain $R_{j}$ is $r_{j}^{*}=\min \left\{\hat{r}_{j}, \bar{r}_{j}\right\} \forall j=1,2, \ldots, N$. Hence, $\mathbf{r}=\left(r_{1}^{*}, \ldots, r_{N}^{*}\right)$ is the unique Nash equilibrium.

A NRPG in transmitted power is defined for each fixed $\mathbf{r} \in R$ as $G(\mathbf{r})=\left[\mathcal{N},\left\{P_{j}\right\},\left\{u_{j}(\mathbf{r},).\right\}\right]$.

A Nash equilibrium in transmitted powers is defined formally as

Definition 2: A power vector $\mathbf{p}=\left(p_{1}, \ldots, p_{N}\right)$ is a Nash equilibrium of the NRPG $G(\mathbf{r})$ if for every $j \in \mathcal{N}$, $u_{j}\left(\mathbf{r}, p_{j}, \mathbf{p}_{-j}\right) \geq u_{j}\left(\mathbf{r}, p_{j} \prime, \mathbf{p}_{-j}\right)$ for all $p_{j} \prime \in P_{j}$, where $\mathbf{p}_{-j}=$ $\left(p_{1}, \ldots, p_{j-1}, p_{j+1}, \ldots, p_{N}\right)^{T}$ is the $N-1$ dimensional vector of user power that does not contain user $j$ 's power.

Theorem 1: For each $\mathbf{r} \in R$, the game $\left[\mathcal{N},\left\{P_{j}\right\},\left\{u_{j}(\mathbf{r},).\right\}\right]$ admits a Nash equilibrium.

Proof: For any fixed $\mathbf{r}$ and $\mathbf{p}_{-j}, u_{j}$ has a unique maximizing point $\hat{p}_{j}$ on $(0, \infty)$ and

(i) $\hat{p}_{j} \in\left(0, \frac{2(M-1) r_{j}}{M c_{j}}\right)$

(ii) $u_{j}$ strictly increases on $\left(0, \hat{p}_{j}\right)$

(iii) $u_{j}$ strictly decreases on $\left(\hat{p}_{j}, \infty\right)$

Since the derivative of $u_{j}$ with respect to $p_{j}$ is

$$
\frac{\partial u_{j}}{\partial p_{j}}=\frac{L}{M p_{j}^{2}}\left(1-e^{-\frac{p_{j} c_{j}}{2 r_{j}}}\right)^{M-1} \psi\left(p_{j}\right)
$$

where

$$
\psi\left(p_{j}\right)=-r_{j}+r_{j} e^{\frac{-p_{j} c_{j}}{2 r_{j}}}+\frac{M p_{j} c_{j}}{2} e^{-\frac{p_{j} c_{j}}{2 r_{j}}}
$$

the proof is analogous to the proof of (a)-(c) in Proposition 1.

Now let us recall the following definition:

Definition 3: A function $f$ is quasi-concave on a convex set $D$ if, for every $\alpha \in \mathbb{R}$, the set $\{x \in D ; f(x) \geq \alpha\}$ is convex.

From (ii) and (iii) it results that all sets $\left\{p_{j} \in\right.$ $\left.(0, \infty) ; u_{j}\left(p_{j}\right) \geq \alpha\right\}$ are intervals, i.e are convex which implies that $u_{j}$ is quasi concave on $(0, \infty)$.

Since the strategy spaces of the game $G(\mathbf{r})$ are not compact, we can not apply directly the equilibrium theorem (Theorem 3.1 and its Corollary) of Nikaido and Isoda [10]. If the strategy spaces are compact convex sets in Euclidean spaces, the player i's utility function is continuous on the product space and quasi-concave with respect to the $i^{\text {th }}$ argument, then the game has Nash equilibria.

However, the game restricted to the strategy spaces $P_{j}^{\prime}=$ $\left[\underline{p}_{j}, \bar{p}_{j}\right], j=1,2, \ldots, N$, admits Nash equilibria in transmission power, for every choice of $\underline{p}_{j}, 0<\underline{p}_{j}<\bar{p}_{j}, j=1,2, \ldots, N$, because it satisfies all assumptions of the theorem of Nikaido and Isoda [10].

Let $p_{j}^{0}=\frac{2 r_{j}}{\bar{c}_{j}}$, where $\bar{c}_{j}=\frac{W h_{j}}{\sigma^{2}}, j=1,2, . ., N$. Then for every $j$ and every $\mathbf{p}_{-j}, B_{j}\left(\mathbf{p}_{-j}\right) \cap\left(0, p_{j}^{0}\right)=\emptyset$, where $B_{j}\left(\mathbf{p}_{-j}\right)$ is the set of all best response strategies of player $j$ against $\mathbf{p}_{-j}$. In other words, there are no equilibrium startegies of user $j$ in the interval $\left(0, p_{j}^{0}\right)$.

To prove this assertion, pick a $j$ and $\mathbf{p}_{-j} \in P_{-j}$. Set $\tilde{p}_{j}=$ $\frac{2 r_{j}}{c_{j}}$. Then $\psi\left(\tilde{p}_{j}\right)=r_{j}\left(-1+e^{-1}+2 M e^{-1}\right)>0$. Hence by (5), $\tilde{p}_{j} \in\left(0, \hat{p}_{j}\right)$. Then, $u_{j}\left(p_{j}, \mathbf{p}_{-j}\right)<u_{j}\left(\hat{p}_{j}, \mathbf{p}_{-j}\right)$, for every $p_{j} \leq \tilde{p}_{j}$. This means that $B_{j}\left(\mathbf{p}_{-j}\right) \cap\left(0, \tilde{p}_{j}\right)=\emptyset$. Since $p_{j}^{0} \leq$ $\tilde{p}_{j}$, the above assertion is proved.

Finally, set $\underline{p}_{j}=\min \left\{p_{j}^{0}, \bar{p}_{j}\right\}, j=1,2, . ., N$ and consider the restricted game with strategy spaces $\left[p_{j}, \bar{p}_{j}\right]$. As it is shown in the above, this game admits Nash equilibria. By the last assertion, every equilibrium of the restricted game is an equilibrium of the original game.

\section{B. The Nash Equilibrium for the Joint Rate and Power Game}

We note that the utility is a function of two variables, rate $r_{j}$ and power $p_{j}$. The existence of equilibrium in transmission rates or powers does not guarantee that there is an equilibrium 
in the joint rate and power control game. Next we prove that there exists a Nash equilibrium in the joint rate and power control game.

Theorem 2: There exists a Nash equilibrium for the game $G=\left[\mathcal{N},\left\{R_{j} \times P_{j}\right\},\left\{u_{j}\right\}\right]$.

Proof: For each $\mathbf{p}$ define the functions $v_{j}, j=1,2, . ., N$, on $(0, \infty)$ by

$$
v_{j}\left(x_{j}\right)=\frac{L}{M} x_{j}\left(1-e^{-\frac{c_{j}}{2 x_{j}}}\right)^{M}
$$

We will write $v_{j}\left(x_{j}, \mathbf{p}_{-j}\right)$ when we need to stress the dependence on $\mathbf{p}_{-j}$ and note that for $(\mathbf{r}, \mathbf{p}) \in R \times P$, $v_{j}\left(\frac{r_{j}}{p_{j}}, \mathbf{p}_{-j}\right)=u(\mathbf{r}, \mathbf{p})$.

The derivative of $v_{j}$ with respect to $x_{j}$, we have

$$
\frac{\partial v_{j}}{\partial x_{j}}=\frac{L}{M}\left(1-e^{-\frac{c_{j}}{2 x_{j}}}\right)^{M-1} \varsigma\left(x_{j}\right)
$$

where

$$
\varsigma\left(x_{j}\right)=1-e^{-\frac{c_{j}}{2 x_{j}}}-\frac{M c_{j}}{2 x_{j}} e^{-\frac{c_{j}}{2 x_{j}}}
$$

As above, we can show that $\lim _{x_{j} \rightarrow 0} \varsigma\left(x_{j}\right)=1$, $\lim _{x_{j} \rightarrow \infty} \varsigma\left(x_{j}\right)=0$ and $\frac{d \varsigma\left(x_{j}\right)}{d x_{j}}$ has a unique zero at $\frac{M c_{j}}{2(M-1)}$ and changes the sign from plus to minus.

It follows that, that for each $\mathbf{p}_{-j} \in P_{-j}, v_{j}$ has a unique maximizing point $\hat{x}_{j}$ on $(0, \infty)$ and

(A) $\hat{x}_{j} \in\left(0, \frac{M c_{j}}{2(M-1)}\right)$

(B) $u_{j}$ strictly increases on $\left(0, \hat{x}_{j}\right)$

(C) $u_{j}$ strictly decreases on $\left(\hat{x}_{j}, \infty\right)$

Now, set $\underline{x}_{j}=\frac{\underline{c}_{j}}{2 M}$ and $\bar{x}_{j}=\frac{\bar{c}_{j}}{2}$, where $\underline{c}_{j}=\frac{W h_{j}}{\sum_{k \neq j} h_{k} \bar{p}_{k}+\sigma^{2}}$ and $\bar{c}_{j}=\frac{W h_{j}}{\sigma^{2}}$.

Then for every $\mathbf{p}$,

$$
\frac{\partial v_{j}}{\partial x_{j}}\left(x_{j}, \mathbf{p}_{-j}\right)>0, \quad \forall x_{j} \in\left(0, \underline{x}_{j}\right)
$$

Therefore $v_{j}$ strictly increases on $\left(0, \underline{x}_{j}\right)$, and

$$
\frac{\partial v_{j}}{\partial x_{j}}\left(x_{j}, \mathbf{p}_{-j}\right)<0, \quad \forall x_{j} \in\left(\bar{x}_{j}, \infty\right)
$$

Therefore $v_{j}$ strictly decreases on $\left(\bar{x}_{j}, \infty\right)$.

Indeed, we have

$$
\varsigma\left(\frac{c_{j}}{2 M}\right)=1-e^{-M}-M^{2} e^{-M}>0
$$

and

$$
\varsigma\left(\frac{c_{j}}{2}\right)=1-(M+1) e^{-1}<0
$$

Then, (B) and (C) imply

$$
\begin{aligned}
& \frac{\partial v_{j}}{\partial x_{j}}\left(x_{j}, \mathbf{p}_{-j}\right)>0, \quad \forall x_{j} \in\left(0, \frac{c_{j}}{2 M}\right) \\
& \frac{\partial v_{j}}{\partial x_{j}}\left(x_{j}, \mathbf{p}_{-j}\right)<0, \quad \forall x_{j} \in\left(\frac{c_{j}}{2}, \infty\right)
\end{aligned}
$$

respectively. Since $\underline{x}_{j} \leq \frac{c_{j}}{2 M}$ and $\bar{x}_{j} \geq \frac{c_{j}}{2}$ for every $\mathbf{p}$, it follows that (15) and (16) hold.

Once again, we use the Nikaido-Isoda theorem for the restricted game $\left[\mathcal{N},\left\{\left[\underline{r}_{j}, \bar{r}_{j}\right] \times\left[\underline{p}_{j}, \bar{p}_{j}\right]\right\},\left\{u_{j}(\cdot, \cdot)\right\}\right]$, where 0 $<\underline{r}_{j} \leq \bar{r}_{j}$ and $0<\underline{p}_{j} \leq \bar{p}_{j}$.
Obviously, the strategy spaces are nonempty, compact and convex and the utility functions are jointly continuous in $\left(r_{j}, p_{j}\right)$.

The shape of $v_{j}$ (as a function of $x_{j}$ ), as it results form (B) and (C) shows that this function is quasi-concave on $(0, \infty)$, more precisely, the upper contour sets of the form $\left\{x_{j} \in(0, \infty) \mid v_{j}\left(x_{j}\right) \geq \alpha\right\}$ are intervals. Now observe that, for any real number $\alpha$, and for any $\mathbf{p}_{-j} \in P_{-j}$,

$$
\begin{aligned}
& \left\{\left(r_{j}, p_{j}\right) \in\left[\underline{r}_{j}, \bar{r}_{j}\right] \times\left[\underline{p}_{j}, \bar{p}_{j}\right] \mid u_{j}\left(r_{j}, p_{j}, p_{-j}\right) \geq \alpha\right\} \\
& =\left\{\left(r_{j}, p_{j}\right) \in\left[\underline{r}_{j}, \bar{r}_{j}\right] \times\left[\underline{p}_{j}, \bar{p}_{j}\right] \mid v_{j}\left(\frac{r_{j}}{p_{j}}, p_{-j}\right) \geq \alpha\right\}
\end{aligned}
$$

Suppose that $\left\{x_{j} \in(0, \infty) \mid v_{j}\left(x_{j}\right) \geq \alpha\right\}=[a, b]$. (The cases when this set is $(0, a)$ or $(b, \infty)$ could also be considered). Then

$$
\begin{aligned}
& \left\{\left(r_{j}, p_{j}\right) \in\left[\underline{r}_{j}, \bar{r}_{j}\right] \times\left[\underline{p}_{j}, \bar{p}_{j}\right] \mid v_{j}\left(\frac{r_{j}}{p_{j}}, p_{-j}\right) \geq \alpha\right\} \\
= & \left\{\left(r_{j}, p_{j}\right) \in\left[\underline{r}_{j}, \bar{r}_{j}\right] \times\left[\underline{p}_{j}, \bar{p}_{j}\right] \mid a p_{j} \leq r_{j} \leq b p_{j}\right\}
\end{aligned}
$$

which is a convex set in $\mathbb{R}^{2}$. Hence, $u_{j}$ is quasi-concave in $\left(r_{j}, p_{j}\right)$.

We can conclude that the restricted game has a Nash equilibrium.

Finally, we will argue that the game $\left[\mathcal{N},\left\{R_{j} \times\right.\right.$ $\left.\left.P_{j}\right\},\left\{u_{j}(\cdot, \cdot)\right\}\right]$ admits a Nash equilibrium.

For each $j$, choose $\underline{r}_{j}, \underline{p}_{j}$ such that $0<\underline{r}_{j} \leq$ $\min \left\{\frac{\underline{c}_{j}}{2 M} \bar{p}_{j}, \bar{r}_{j}\right\}, 0<\underline{p}_{j} \leq \min \left\{\frac{2 \bar{r}_{j}}{\bar{c}_{j}}, \bar{p}_{j}\right\}$. By the above, it results that the restricted game $\left[\mathcal{N},\left\{\left[\underline{r}_{j}, \bar{r}_{j}\right] \times\left[\underline{p}_{j}, \bar{p}_{j}\right]\right\},\left\{u_{j}(\cdot, \cdot)\right\}\right]$ has equilibria. Let $\left(r^{*}, p^{*}\right)$ be a Nash equilibrium of this game. Then, for every $j$,

$u_{j}\left(r_{j}, p_{j}, \mathbf{p}_{-j}^{*}\right) \leq u_{j}\left(\mathbf{r}^{*}, \mathbf{p}^{*}\right), \quad \forall\left(r_{j}, p_{j}\right) \in\left[\underline{r}_{j}, \bar{r}_{j}\right] \times\left[\underline{p}_{j}, \bar{p}_{j}\right]$

Next, we will prove that $\left(\mathbf{r}^{*}, \mathbf{p}^{*}\right)$ is an equilibrium of the game $\left[\mathcal{N},\left\{R_{j} \times P_{j}\right\},\left\{u_{j}(\cdot, \cdot)\right\}\right]$.

Pick a $j$ and $\left(r_{j}, p_{j}\right) \in R_{j} \times P_{j}$ and denote $x_{j}=\frac{r_{j}}{p_{j}}$. Three possible situations will be considered:
(I) $x_{j} \in\left[\frac{\underline{r}_{j}}{\bar{p}_{j}}, \frac{\bar{r}_{j}}{\underline{p}_{j}}\right]$
(II) $x_{j}<\frac{\underline{r}_{j}}{\bar{p}_{j}}$
(III) $x_{j}>\frac{\bar{r}_{j}^{j}}{\underline{p}_{j}}$

In case (I) we show that there exists $\left(r_{j}^{\prime}, p_{j}^{\prime}\right) \in\left[\underline{r}_{j}, \bar{r}_{j}\right] \times$ $\left[\underline{p}_{j}, \bar{p}_{j}\right]$ such that $x_{j}=\frac{r_{j}^{\prime}}{p_{j}^{\prime}}$.

Obviously, since $x_{j} \in\left[\frac{\underline{\underline{p}}_{j}}{\bar{p}_{j}}, \frac{\bar{r}_{j}}{\underline{p}_{j}}\right]$, then either $x_{j} \in\left[\frac{\underline{r}_{j}}{\bar{p}_{j}}, \frac{\bar{r}_{j}}{\bar{p}_{j}}\right]$, or $x_{j} \in\left[\frac{\bar{r}_{j}}{\bar{p}_{j}}, \frac{\bar{r}_{j}}{\underline{p}_{j}}\right]$.

In the first case take $r_{j}^{\prime}=x_{j} \bar{p}_{j}$ and $p_{j}^{\prime}=\bar{p}_{j} \cdot\left(r_{j}^{\prime} \geq \frac{\underline{r}_{j}}{\bar{p}_{j}} \bar{p}_{j}=\right.$ $\underline{r}_{j}, r_{j}^{\prime} \leq \overline{\bar{r}}_{j} \bar{p}_{j}=\bar{r}_{j}$ )

In the second case take $r_{j}^{\prime}=\bar{r}_{j}$ and $p_{j}^{\prime}=\frac{\bar{r}_{j}}{x_{j}} \cdot\left(p_{j}^{\prime} \geq \bar{r}_{j} \frac{\underline{r}_{j}}{\bar{r}_{j}}=\right.$ $\left.\underline{p}_{j}, p_{j}^{\prime} \leq \bar{r}_{j} \frac{\bar{p}_{j}}{\bar{r}_{j}}=\bar{p}_{j}\right)$

Then, by (21)

$$
\begin{aligned}
u_{j}\left(r_{j}, p_{j}, \mathbf{p}_{-j}^{*}\right) & =v_{j}\left(x_{j}, \mathbf{p}_{-j}^{*}\right)=v_{j}\left(\frac{r_{j}^{\prime}}{p_{j}^{\prime}}, \mathbf{p}_{-j}^{*}\right) \\
& =u_{j}\left(r_{j}^{\prime}, p_{j}^{\prime}, \mathbf{p}_{-j}^{*}\right) \leq u_{j}\left(r_{j}^{*}, p_{j}^{*}\right)
\end{aligned}
$$


In case (II), $x<\frac{\underline{c}_{j}}{2 M}=\underline{x}_{j}$ and by (15) and (21) we have

$$
\begin{aligned}
u_{j}\left(r_{j}, p_{j}, \mathbf{p}_{-j}^{*}\right) & =v_{j}\left(x_{j}, \mathbf{p}_{-j}^{*}\right)<v_{j}\left(\frac{\underline{r}_{j}}{\bar{p}_{j}}, \mathbf{p}_{-j}^{*}\right) \\
= & u_{j}\left(\underline{r}_{j}, \bar{p}_{j}, \mathbf{p}_{-j}^{*}\right) \leq u_{j}\left(r_{j}^{*}, p_{j}^{*}\right)
\end{aligned}
$$

Similarly, in case (III), since $x_{j}>\frac{\bar{c}_{j}}{2}=\bar{x}_{j}$, it follows from (16) and (21) that

$$
\begin{aligned}
u_{j}\left(r_{j}, p_{j}, \mathbf{p}_{-j}^{*}\right) & =v_{j}\left(x_{j}, \mathbf{p}_{-j}^{*}\right)<v_{j}\left(\frac{\bar{r}_{j}}{\underline{p}_{j}}, \mathbf{p}_{-j}^{*}\right) \\
& =u_{j}\left(\bar{r}_{j}, \underline{p}_{j}, \mathbf{p}_{-j}^{*}\right) \leq u_{j}\left(r_{j}^{*}, p_{j}^{*}\right)
\end{aligned}
$$

\section{The Joint Rate And Power Control Algorithm}

In the proposed NRPG algorithm for joint rate and power control, the users update their rates and powers asynchronously such that for a given user the new rate and power values are computed in the same step. Note that, as shown in the proof of Theorem 3, for finding a Nash equilibrium of the game $\left[\mathcal{N},\left\{R_{j} \times P_{j}\right\},\left\{u_{j}(\cdot, \cdot)\right\}\right]$, it is enough to consider the restricted game $\left[\mathcal{N},\left\{\left[\underline{r}_{j}, \bar{r}_{j}\right] \times\left[\underline{p}_{j}, \bar{p}_{j}\right]\right\},\left\{u_{j}(\cdot, \cdot)\right\}\right]$, where $\underline{r}_{j}$ and $\underline{p}_{j}$ are chosen as in Theorem 2 . The joint rate and power control algorithm based on NRPG is formally stated below:

\section{NRPG Algorithm}

(1) Initialize the rates $\mathbf{r}\left(t_{0}\right)=\left[r_{1}\left(t_{0}\right), \ldots, r_{N}\left(t_{0}\right)\right]$ and power vector $\mathbf{p}\left(t_{0}\right)=\left[p_{1}\left(t_{0}\right), \ldots, p_{N}\left(t_{0}\right)\right]$ at time $t_{0}$ with $r_{j}\left(t_{0}\right)=\bar{r}_{j}$ and $p_{j}\left(t_{0}\right)=p_{j}, j=1,2, \ldots, N$. Each user $j(j \in \mathcal{N})$ at time instant $t_{k}$ computes:

a)

$$
\begin{aligned}
& \text { Compute } x_{j}\left(t_{k}\right), \text { for } t_{k} \geq t_{0} \text {, as } \\
& \qquad x_{j}\left(t_{k}\right)=\arg \max _{x_{j} \in\left[\frac{\underline{r}_{j}}{\bar{p}_{j}}, \frac{\bar{r}_{j}}{\underline{\underline{p}}_{j}}\right]} v_{j}\left(x, \mathbf{p}_{-j}\left(t_{k}\right)\right)
\end{aligned}
$$

b) Evaluate the rates $r_{j}\left(t_{k+1}\right)$ and powers $p_{j}\left(t_{k+1}\right)$ as

$$
\left(r_{j}\left(t_{k+1}\right), p_{j}\left(t_{k+1}\right)\right)=\left\{\begin{array}{l}
\left(x_{j}\left(t_{k}\right) \bar{p}_{j}, \bar{p}_{j}\right), \\
\text { if } x_{j}\left(t_{k}\right) \leq \bar{r}_{j} \\
\left(\bar{p}_{j}, \frac{\bar{r}_{j}}{x_{j}\left(t_{k}\right)}\right), \\
\text { if } x_{j}\left(t_{k}\right)>\frac{\bar{r}_{j}}{\bar{p}_{j}}
\end{array}\right.
$$

If $\max \left\{\left\|\mathbf{r}\left(t_{k}\right)-\mathbf{r}\left(t_{k+1}\right)\right\|,\left\|\mathbf{p}\left(t_{k}\right)-\mathbf{p}\left(t_{k+1}\right)\right\|\right\} \leq \epsilon$, then STOP. Else, make $k=k+1$ and go to step (2).

The power and rate update equations require the knowledge of the interference plus noise experienced by user $j$ 's signal at the base station i.e.

$$
\left(\sum_{k=1, j \neq k}^{N} h_{k} p_{k}+\sigma^{2}\right)
$$

It is assumed that the base station broadcasts the "total power plus noise" term $\left(\sum_{k=1}^{N} h_{k} p_{k}+\sigma^{2}\right)$ to all the users. In addition, assuming reciprocal communication channels each user $j$ knows its channel gain to the base station $h_{j}$ and can estimate their received power $h_{j} p_{j}$ at the base station which is subtracted from the "total power plus noise" term to obtain the term in (25) needed for updating transmission rates and powers.

Theorem 3: For each $j$, the sequence $r_{j}(t)$ decreases and converges to a limit $r_{j}^{*}$, and the sequence $p_{j}(t)$ increases and converges to a limit $p_{j}^{*}$. The pair $\left(\mathbf{r}^{*}, \mathbf{p}^{*}\right)$ is a Nash equilibrium of the game.

Proof: First recall that, for each $\mathbf{p}_{-j}$, the function $v_{j}\left(\cdot, \mathbf{p}_{-j}\right)$ has a unique maximizing point on $(0, \infty)$, denoted by $\hat{x}_{j}$, and this is the unique root of the equation (14). As $\hat{x}_{j}$ depends on $\mathbf{p}$, this dependence can be expressed as the dependence on $c_{j}$. Therefore, considering $\varsigma\left(x_{j}\right)$ as a function of $\left(x_{j}, c_{j}\right)$, the equation $\varsigma\left(x_{j}, c_{j}\right)=0$ defines implicitly $\hat{x}_{j}$ as a function of $c_{j}, \hat{x}_{j}=\hat{x}_{j}\left(c_{j}\right)$. The implicit function theorem says that

$$
\frac{\partial \hat{x}_{j}}{\partial c_{j}}=-\frac{\frac{\partial \varsigma}{\partial c_{j}}}{\frac{\partial \varsigma}{\partial x_{j}}}
$$

which by a simple calculation gives us $\frac{\partial \hat{x}_{j}}{\partial c_{j}}=\frac{x_{j}}{c_{j}}>0$, i.e. $\hat{x}_{j}$ strictly increases with $c_{j}$.

Now, we can prove the first part of the theorem by induction on $t_{k}$.

It is obvious that, for each $j, r_{j}\left(t_{1}\right) \leq r_{j}\left(t_{0}\right)=\bar{r}_{j}$ and $p_{j}\left(t_{1}\right) \geq p_{j}\left(t_{0}\right)=\underline{p}_{j}$.

Now, suppose that $r_{j}\left(t_{k}\right) \leq r_{j}\left(t_{k-1}\right)$ and $p_{j}\left(t_{k}\right) \geq$ $p_{j}\left(t_{k-1}\right)$ for some $k \geq 1$ and for every $j$. Then, for each $j, c_{j}\left(t_{k}\right) \leq c_{j}\left(t_{k-1}\right)$ and since

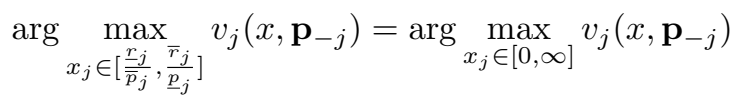

it follows from the above that $x_{j}\left(t_{k}\right) \leq x_{j}\left(t_{k-1}\right)$.

This means that, in the NRPG algorithm at the Step (2), b) at time $t_{k+1}$, either the user switches strategy from case (II) to case (I) (step (2) b) of NRPG algorithm) or the user retains the strategy of case (II) but increases $p_{j}$ and decreases $r_{j}$. In both situations $r_{j}\left(t_{k}\right) \leq r_{j}\left(t_{k-1}\right)$ and $p_{j}\left(t_{k}\right) \geq p_{j}\left(t_{k-1}\right)$.

Since the sequences $r_{j}\left(t_{k}\right)$ and $p_{j}\left(t_{k}\right)$ are monotone in bounded intervals, they are convergent.

(I) Let $r_{j}^{*}$ and $p_{j}^{*}$, respectively be the limits of these sequences. Now, since $x_{j}\left(t_{k}\right)=\frac{r_{j}\left(t_{k+1}\right)}{p_{j}\left(t_{k+1}\right)}$, it follows that

(II)

$$
\begin{gathered}
u_{j}\left(r_{j}\left(t_{k+1}\right), p_{j}\left(t_{k+1}\right), \mathbf{p}_{-j}\left(t_{k}\right)\right)=v_{j}\left(x_{j}\left(t_{k}\right), \mathbf{p}_{-j}\left(t_{k}\right)\right) \\
\geq v_{j}\left(x, \mathbf{p}_{-j}\left(t_{k}\right)\right), \forall x \in\left[\frac{\underline{r}_{j}}{\bar{p}_{j}}, \frac{\bar{r}_{j}}{\underline{p}_{j}}\right]
\end{gathered}
$$

Now, pick an arbitrary $\left(r_{j}, p_{j}\right) \in\left[\underline{r}_{j}, \bar{r}_{j}\right] \times\left[\underline{p}_{j}, \bar{p}_{j}\right]$. Then, $\frac{r_{j}}{p_{j}} \in\left[\frac{\underline{r}_{j}}{\bar{p}_{j}}, \frac{\bar{r}_{j}}{\underline{p}_{j}}\right]$, so that, the above inequality implies that

$$
\begin{gathered}
u_{j}\left(r_{j}\left(t_{k+1}\right), p_{j}\left(t_{k+1}\right), \mathbf{p}_{-j}\left(t_{k}\right)\right) \geq \\
v_{j}\left(\frac{r_{j}}{p_{j}}, \mathbf{p}_{-j}\left(t_{k}\right)\right)=u_{j}\left(r_{j}, p_{j}, \mathbf{p}_{-j}\left(t_{k}\right)\right)
\end{gathered}
$$

By continuity of $u_{j}$, the inequality between the extreme terms yields

$$
u_{j}\left(r_{j}^{*}, p_{j}^{*}, \mathbf{p}_{-j}^{*}\right) \geq u_{j}\left(r_{j}, p_{j}, \mathbf{p}_{-j}^{*}\right)
$$


Theorem 3 proves that for the choice of initial rates and power values specified in Step (1) of the NRPG algorithm this will always reach the same Nash equilibrium point characterized by the pair $\left(\mathbf{r}^{*}, \mathbf{p}^{*}\right)$ where the individual elements $r_{j}^{*}$ in $\mathbf{r}^{*}$ and $p_{j}^{*}$ in $\mathbf{p}^{*}$ contain the limits of the sequences $r_{j}(t)$ and $p_{j}(t)$ which are monotonically decreasing and increasing, respectively for all $j$. Thus, even though many Nash equilibria for the NRPG game may be possible depending on the initial strategies of the players, the NRPG algorithm with the initialization specified in Step (1) will always converge to the same Nash equilibrium point and from this perspective the outcome of the NRPG algorithm is unique. We note that, from a practical perspective, all users are interested in transmitting at highest rate possible with lowest required power, and in the context of Theorem 3 initializing user powers with the minimum value in the power strategy space and user rates with the maximum value in the rate strategy space is a rational choice.

\section{Simulation Setup and Numerical Results}

We performed simulations to study the NRPG algorithm in a single cell CDMA system and compared it with the rate and power control algorithm described in [15] which we refer to as the 'ZL algorithm' (or simply 'ZL'). The ZL algorithm initializes all user rates and powers with zero, and in one step of the algorithm each user updates rate and power with the corresponding solutions of a nonlinear programming problem with two variables that maximize the user payoff function. A formal statement of the ZL algorithm is given in [15] and we refer readers to [15] for specific details about it.

The parameters of the simulation setup were chosen similar to [15] and these are:

- All users are assumed to be stationary, and the propagation model has channel gains $h_{j}=c / d_{j}^{4}$, where $d_{j}$ is the distance (in meters) between user $j$ and the base station and $c=0.097$.

- The power spectral density of the AWGN at the receiver is $\sigma^{2}=5 \times 10^{-15} \mathrm{~W} / \mathrm{Hz}$.

- The minimum and maximum powers of each user are $p=\hat{p}=10^{-6} \mathrm{~W}$, respectively $\bar{p}=0.2 \mathrm{~W}$.

- The minimum and maximum transmission rates of each user are $\underline{r}=0.1$ and $\bar{r}=96,000$ [bits/sec] respectively.

The NRPG algorithm is compared to the ZL algorithm for different available bandwidths by considering the location of the users around the base station as shown in Figure 3. The results are shown in Figure 4. When available bandwidth is $W=3.84 \times 10^{6} \mathrm{~Hz}$ as in the numerical examples in [15] the NRPG and the ZL algorithms result in similar powers and rates, with users closer to the base station transmitting at higher rates and lower powers than users farther away from the base station. For both algorithms, as the available bandwidth $W$ is increased the number of users that transmit at the maximum allowed power $\bar{p}$ and/or minimum allowed rate $\underline{r}$ decreases, and the NRPG algorithm results in more efficient power and rate allocations than the $\mathrm{ZL}$ algorithm. As seen in Figure 4(a), when the available bandwidth is increased by about $40 \%$ to $W=5.4 \times 10^{6} \mathrm{~Hz}$ the power

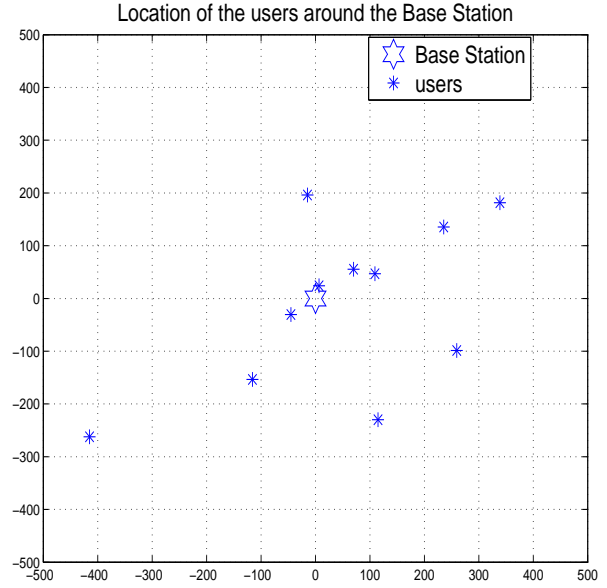

Fig. 3. Example of random locations of users around the Base station

values for users that are transmitting below the maximum power level are about $40 \%$ less for the NRPG algorithm than for the ZL algorithm, and when the available bandwidth is doubled, to $W=7.68 \times 10^{6} \mathrm{~Hz}$, these powers for the NRPG are about half of the corresponding ZL powers. In terms of rates, shown in Figure 4(b), when the bandwidth is increased to $W=5.4 \times 10^{6} \mathrm{~Hz}$ the rate values for users that are transmitting at rates between the minimum rate $\underline{r}$ and maximum rate $\bar{r}$ are approximately 10-20\% higher for the NRPG algorithm than for the ZL algorithm, and when the bandwidth is $W=7.68 \times 10^{6} \mathrm{~Hz}$ the rates for the NRPG are about $25 \%$ to $50 \%$ higher than the corresponding ZL rates. In addition, as seen in Figure 4(c) the NRPG algorithm results always in the equal SIR values for all users as opposed to the $\mathrm{ZL}$ algorithms where users that are closer to the base station get slightly higher SIR values.

In order to corroborate these results and evaluate the average improvements implied by the NRPG algorithm over the ZL algorithm we also performed Monte Carlo simulations and ran 100 trials of both algorithms, each trial with a different random placement of the users around the base station. Results of these simulations are shown in Figures 5-7 and confirm that the NRPG algorithm yields more efficient power and rate allocations than the ZL algorithm: when the available bandwidth is increased average power values (over the 100 simulations) for users that are transmitting below the maximum power level are lower for the NRPG algorithm than for ZL algorithm (Figure 5 ) and average rate values (over the 100 simulations) for users that are transmitting at rates between the minimum and maximum values are higher for the NRPG algorithm than for the ZL algorithm (Figure 6). From Figure 7, we note that the average SIR values (over the 100 simulations) are similar for the NRPG algorithm while for the ZL algorithm the average SIR values for users that are closer to the base station are slightly higher than those of the rest of the users.

Monte Carlo simulations were also used to investigate convergence speed of the NRPG and ZL algorithms, and histograms with the number of steps for convergence to Nash equilibrium in the 100 trials simulated are shown in Figure 8. We note that the NRPG algorithm takes around $20-60$ iterations to converge to the equilibrium (Figure 8(a)) while 


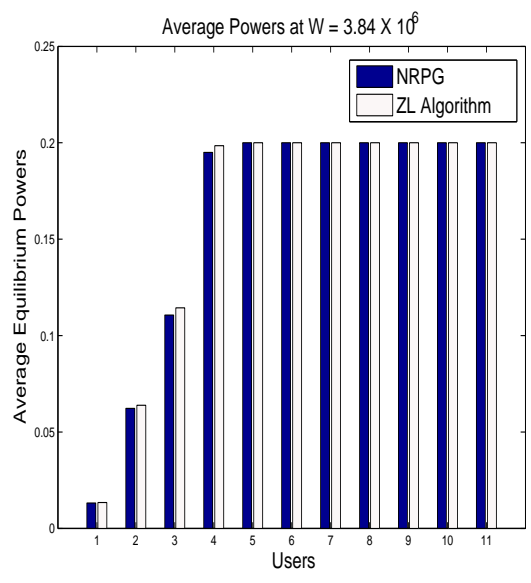

(a) $\mathrm{W}=3.84 \times 10^{6}$

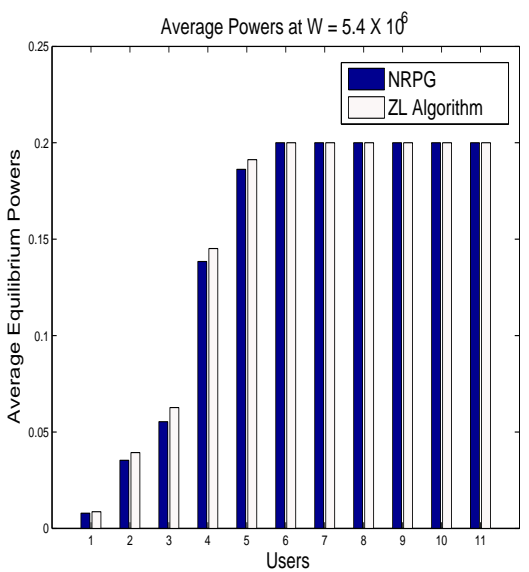

(b) $\mathrm{W}=5.4 \times 10^{6}$

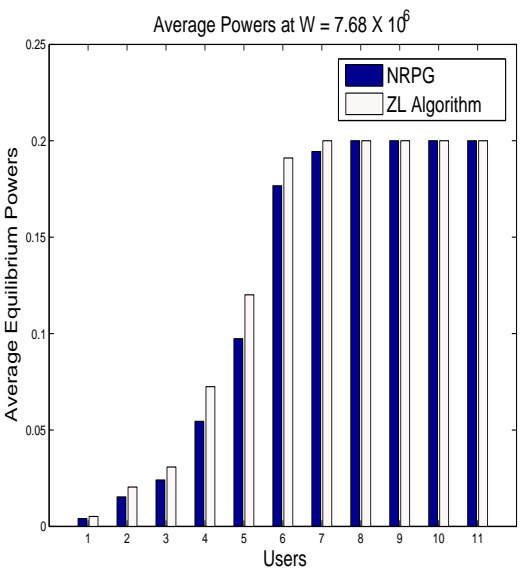

(c) $\mathrm{W}=7.68 \times 10^{6}$

Fig. 5. The average powers yielded by the ZL algorithm and NRPG for a Monte Carlo simulation of 100 runs. User 1 is the closest to the base station and user 11 is the farthest.

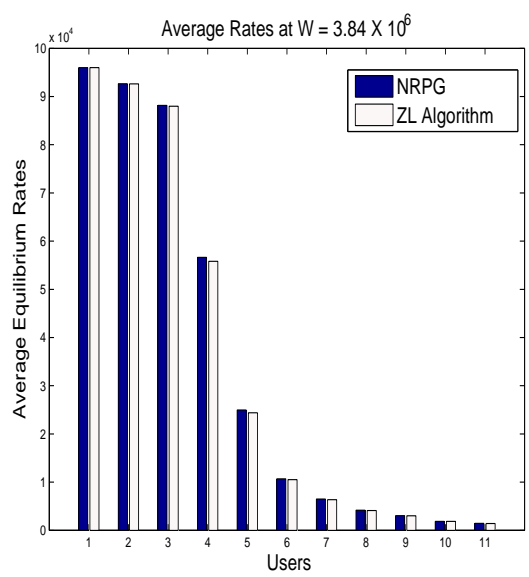

(a) $\mathrm{W}=3.84 \times 10^{6}$

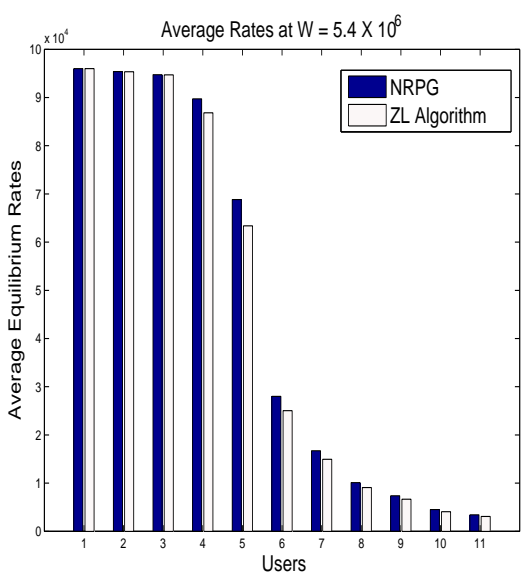

(b) $\mathrm{W}=5.4 \times 10^{6}$

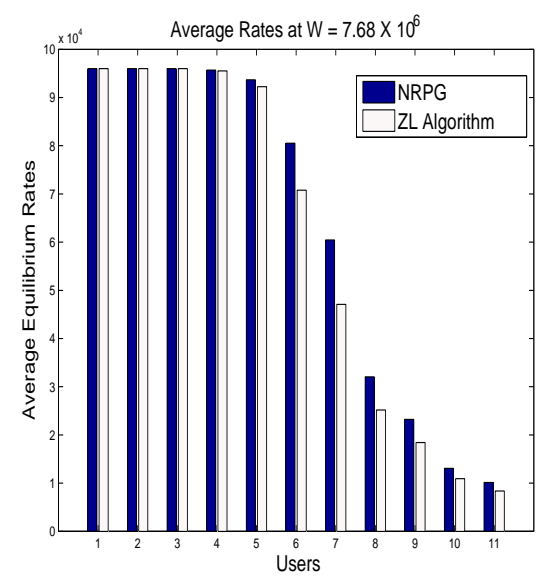

(c) $\mathrm{W}=7.68 \times 10^{6}$

Fig. 6. The average rates yielded by the ZL algorithm and NRPG for a Monte Carlo simulation of 100 runs. User 1 is the closest to the base station and user 11 is the farthest.

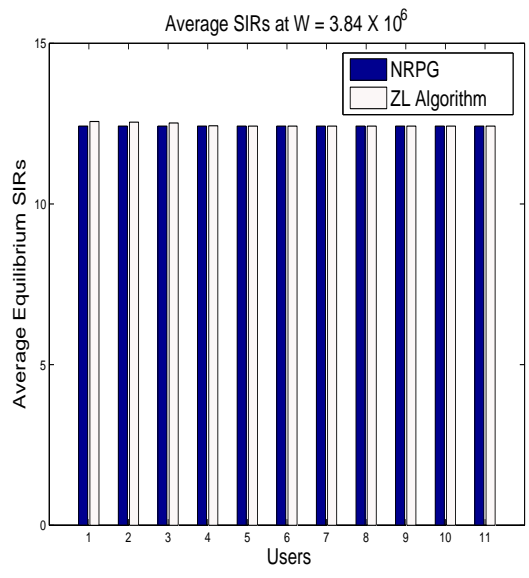

(a) $\mathrm{W}=3.84 \times 10^{6}$

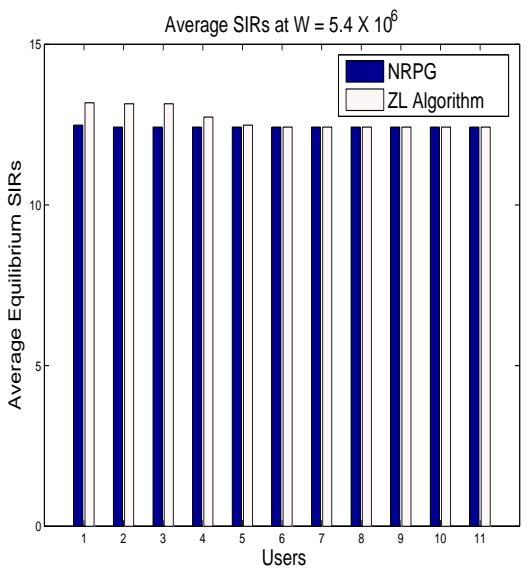

(b) $\mathrm{W}=5.4 \times 10^{6}$

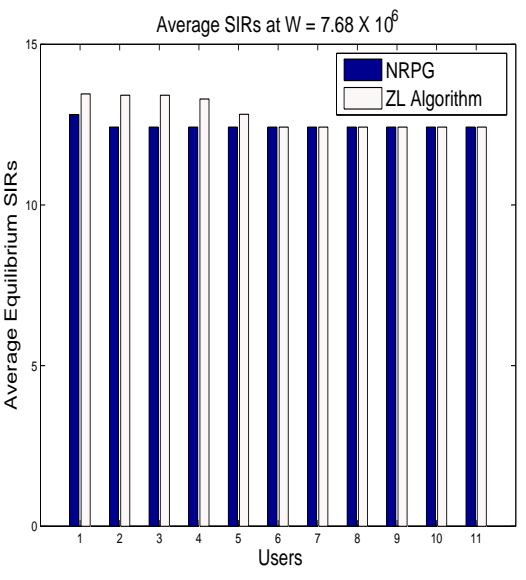

(c) $\mathrm{W}=7.68 \times 10^{6}$

Fig. 7. The average SIRs yielded by ZL algorithm and NRPG for a Monte Carlo simulation of 100 runs. User 1 is the closest to the base station and user 11 is the farthest. 


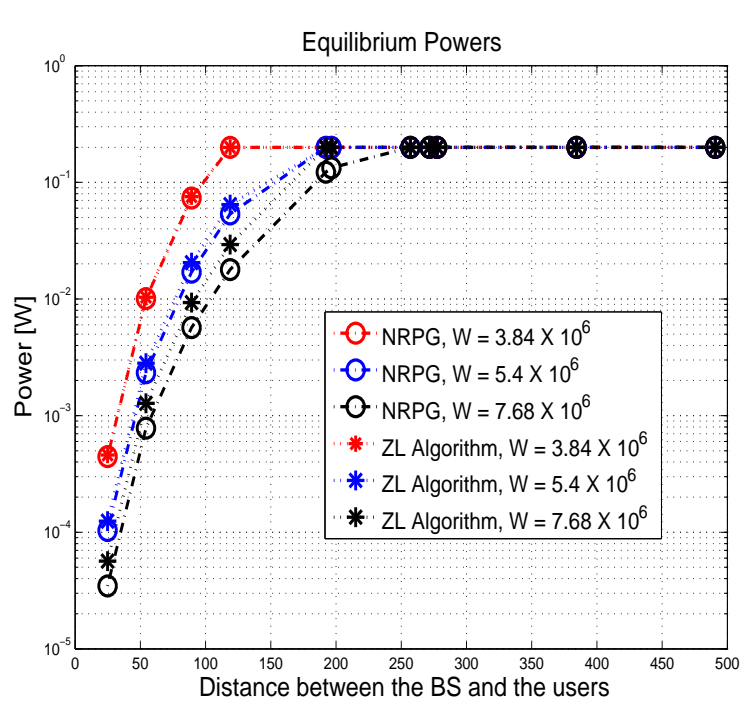

(a) Powers

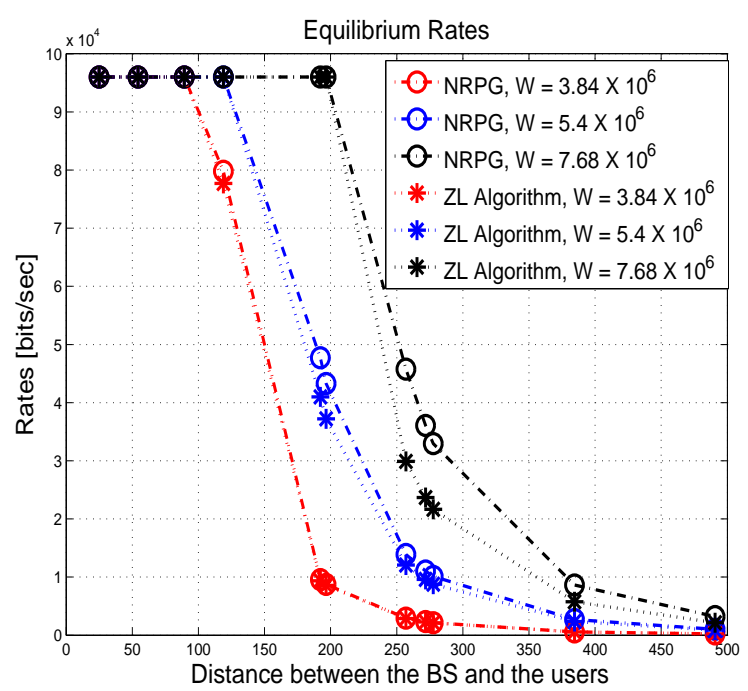

(b) Rates

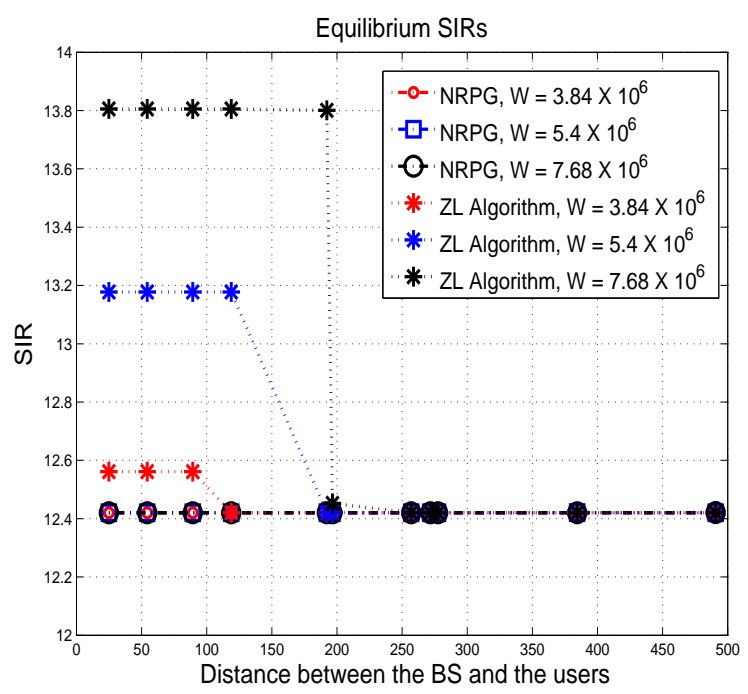

(c) SIRs

Fig. 4. Comparison of user powers, rates and SIRs yielded by the ZL algorithm ( [15]) with those yielded by the NRPG for different bandwidths

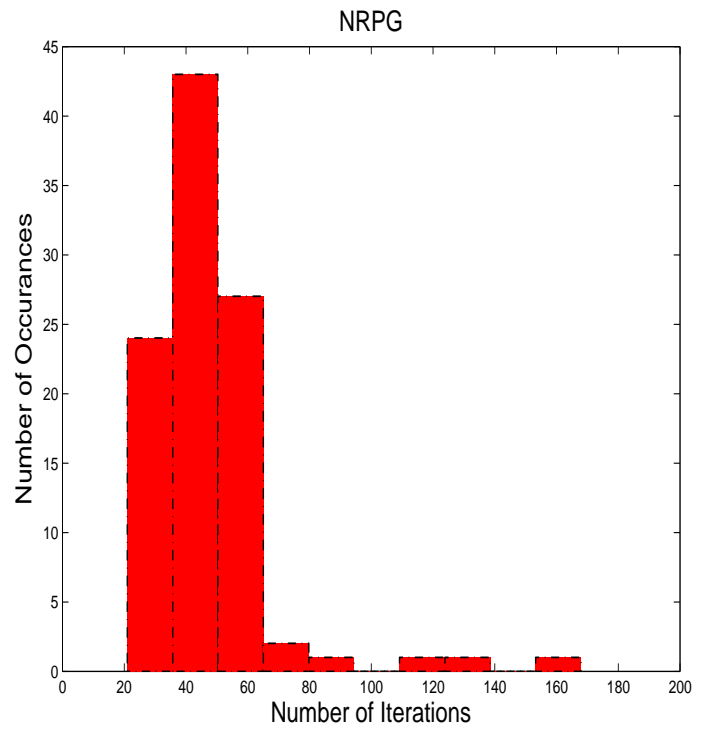

(a) NRPG

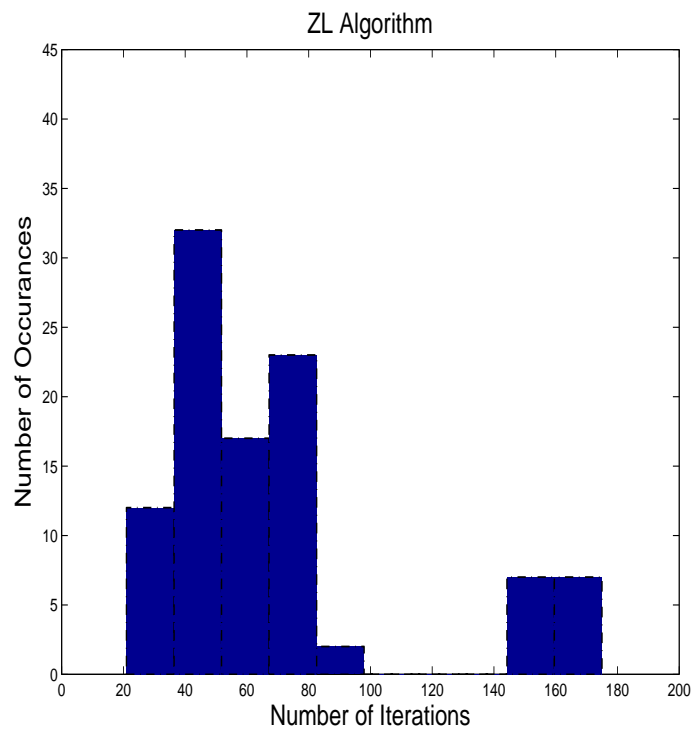

(b) ZL algorithm

Fig. 8. Monte Carlo simulations to investigate convergence of the NRPG and $\mathrm{ZL}$ algorithm

the ZL algorithm takes $40-80$ iterations (Figure $8(\mathrm{~b})$ ). Thus, in addition to the more efficient power and rate allocations noted above the NRPG algorithm is also faster than the ZL algorithm.

\section{CONClusions AND Future Work}

In this paper we applied game theory to model the problem of joint transmission rate and power control for the uplink of a single cell CDMA system as a non-cooperative game. Similar to other approaches to joint rate and power control a static scenario with no users leaving or new users joining the system was considered. The utility function used in our approach is defined by the ratio of throughput to the transmit power and its maximization implies optimal transmission rate and power. We showed the existence of Nash equilibria for 
the joint rate and power control game and formally stated the NRPG algorithm for joint rate and power control.

Numerical simulations were performed to illustrate the proposed NRPG algorithm and to compare it with the ZL algorithm for joint rate and power control developed using a related game-theoretic approach in [15]. While both algorithms work similarly and update user rates and powers simultaneously in one step, simulation results have shown that the NRPG algorithm converges faster than the $\mathrm{ZL}$ algorithm and results in more efficient power and rate allocations. A comparison of the Nash equilibrium point implied by the NRPG algorithm with the optimal power and rate allocations implied by centralized schemes (such as those in [6], [11] for example) will be the object of future work.

\section{ACKNOWLEDGMENT}

The reviewers comments helped improve the quality of presentation of the paper and are gratefully acknowledged.

\section{REFERENCES}

[1] T. Alpcan, T. Basar, and S. Dey, "A power control game based on outage probabilities for multicell wireless data networks," IEEE Transactions on Wireless Communications, vol. 5, no. 4, pp. 890 - 899, April 2006.

[2] S. Ginde, A. MacKenzie, R. Buehrer, and R. S. Komali, "A GameTheoretic Analysis of Link Adaptation in Cellular Radio Networks," IEEE Transactions on Vehicular Technology, vol. 57, no. 5, pp. 31083120, September 2008.

[3] D. J. Goodman and N. B. Mandayam, "Power Control for Wireless Data," IEEE Personal Communications Magazine, vol. 7, no. 2, pp. 4854, April 2000.

[4] S. Gunturi and F. Paganini, "A Game Theoretic Approach to Power Control in Cellular CDMA," in Proceedings $58^{\text {th }}$ IEEE Vehicular Technology Conference - VTC 2003 Fall, vol. 3, Orlando, FL, October 2003, pp. 2362-2366.

[5] M. Hayajneh and C. Abdallah, "Distributed joint rate and power control game-theoretic algorithms for wireless data," IEEE Communications Letters, vol. 8, no. 8, pp. 511-513, August 2004.

[6] S. Jafar and A. Goldsmith, "Adaptive multirate CDMA for uplink throughput maximization ," IEEE Transactions on Wireless Communications, vol. 2, no. 2, pp. 218 - 228, March 2003.

[7] S. Koskie and Z. Gajic, "A nash game algorithm for SIR-based power control in 3G wireless CDMA networks," IEEE/ACM Transactions on Networking, vol. 13, no. 5, pp. 1017 - 1026, October 2005.

[8] C. Long, Q. Zhang, B. Li, H. Yang, and X. Guan, "Non-Cooperative Power Control for Wireless Ad Hoc Networks with Repeated Games," IEEE Journal on Selected Areas in Communications, vol. 25, no. 6, pp. 1101-1112, August 2007.

[9] F. Meshkati, H. Poor, S. Schwartz, and N. Mandayam, "An energyefficient approach to power control and receiver design in wireless data networks," IEEE Transactions on Communications, vol. 53, no. 11, pp. 1885 - 1894, November 2005.

[10] H. Nikaido and K. K. Isoda, "Note on noncooperative convex games." Pacific Journal of Mathematics, vol. 5, pp. 807 - 815, 1955.

[11] D. ONeill, D. Julian, and S. Boyd, "Seeking foschini's genie: Optimal rates and powers in wireless networks," 2003. [Online]. Available: citeseer.ist.psu.edu/oneill03seeking.html

[12] M. J. Osborne, An Introduction to Game Theory. New York, NY: Oxford University Press, 2004.

[13] C. U. Saraydar, N. B. Mandayam, and D. J. Goodman, "Efficient Power Control via Pricing in Wireless Data Networks," IEEE Transactions on Communications, vol. 50, no. 2, pp. 291-303, February 2002.

[14] Z. Yin, J. Xie, and P. Lv, "An Economic Model for Power Control in Wireless CDMA Data Networks," in Proceedings 2003 International Conference on Communication Technology - ICCT 2003, vol. 2, Beijing, People's Republic of China, April 2003, pp. 835-838.

[15] W. Zhao and M. Lu, "Distributed rate and power control for CDMA uplink," in Proceedings 2004 Wireless Telecommunications Symposium, May 2004, pp. $9-14$.
[16] L. Zhu and S. Wu, "Power control for CDMA data communication systems ," in Proceedings 2002 IEEE International Conference on Communications, circuits and Systems and West Sino Expositions, vol. 1, Chengdu, People's Republic of China, June, July 2002, pp. 367-371.

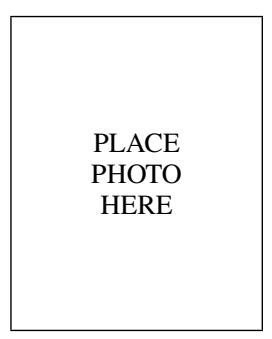

\section{Madhusudhan R. Musku}

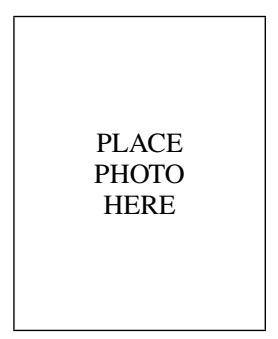

Anthony T. Chronopoulos received his Ph.D. in Computer Science from the University of Illinois at Urbana-Champaign in 1987. He is currently a Professor in Computer Science at the University of Texas at San Antonio. He has published 45 journal and 58 refereed conference proceedings publications in the areas of distributed systems, high performance computing and applications. He has been awarded 15 federal/state government research grants. His work is cited in over 400 non-coauthors' research articles. $\mathrm{He}$ is a senior member of IEEE (since 1997).

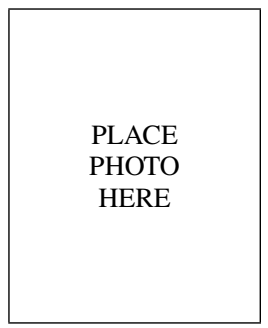

Dimitrie C. Popescu received the Engineering Diploma and M.S. degrees in 1991 from the Polytechnic Institute of Bucharest, Romania, and the Ph.D. degree from Rutgers University in 2002, all in Electrical Engineering. His research interests are in the areas of wireless communications, digital signal processing, and control theory. $\mathrm{He}$ is currently an Assistant Professor in the Department of Electrical and Computer Engineering, Old Dominion University. Between 2002 and 2006 he was with the Department of Electrical and Computer Engineering, the University of Texas at San Antonio. He has also worked for AT\&T Labs in Florham Park, New Jersey, on signal processing algorithms for speech enhancement, and for Telcordia Technologies in Red Bank, New Jersey, on wideband CDMA systems. He has served as technical program chair for the vehicular communications track of the IEEE VTC 2009 Fall, finance chair for the IEEE MSC 2008, and technical program committee member for the IEEE GLOBECOM, WCNC 2006, and VTC conferences. His work on interference avoidance and dispersive channels was awarded second prize in the AT\&T Student Research Symposium in 1999. He is the co-author of a monograph book on interference avoidance methods for wireless systems published in 2004 by Kluwer Academic Publishers.

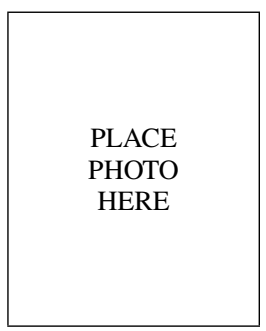

